\title{
Tensor coding for three-hop MIMO relay systems
}

\author{
Danilo S. Rocha \\ Department of Teleinformatics \\ Engineering \\ Federal University of Ceará (UFC) \\ Fortaleza, Brazil \\ danilo@fisica.ufc.br
}

\author{
Gérard Favier \\ I3S Laboratory, CNRS \\ University of Côte d'Azur (UCA) \\ Sophia Antipolis, France \\ favier@i3s.unice.fr
}

\author{
C. Alexandre R. Fernandes \\ Department of Teleinformatics \\ Engineering \\ Federal University of Ceará (UFC) \\ Fortaleza, Brazil \\ alexandrefernandes@ufc.br
}

\begin{abstract}
A three-hop AF MIMO relay system with tensor coding at the source and the relays is considered in this paper. The signals received at destination form a fifth-order tensor that satisfies a high-order nested Tucker decomposition, characterized by the concatenation of three Tucker models. We propose a receiver based on an alternating least square algorithm to jointly estimate the symbol matrix and the channels of each hop. Monte Carlo simulation results are provided to illustrate the behavior of the proposed system and of the semi-blind receiver. These simulation results show a performance closest to the one of the zero-forcing receiver, yielding a significant SER improvement due to the relay-assisted link when compared to the direct link.
\end{abstract}

Keywords-MIMO systems; nested Tucker decomposition; semiblind receivers; tensor coding; tensor models; three-hop relaying.

\section{INTRODUCTION}

The exploitation of multiple-input multiple-output (MIMO) relay systems has been prominent in the development of new signal processing techniques for $5 \mathrm{G}$ communication systems. In the last two decades, the application of tensor models to wireless systems [1]-[2] has allowed the processing of multimodal signals exploiting several diversities like space, time and coding diversities [3]-[6]. In addition, tensor models have interesting uniqueness properties that allow the design of semi-blind receivers, presenting advantages over other approaches that require the use of training sequences [7]-[8].

In the context of cooperative networks [5]-[6],[9]-[10], the use of tensor coding at the transmitting nodes and an amplifyand-forward (AF) relaying protocol (where the relay retransmits data without decoding) leads to received signals that form highorder tensors. A tensor space-time coding (TSTC) was introduced in [3] and applied to a non-cooperative MIMO system, allowing spreading and multiplexing the transmitted symbols, in both space and time domains. In [5], TSTC at the source and the relay was applied to a two-hop MIMO relay system whose signals received at destination satisfy a fourthorder tensor model based on a nested Tucker decomposition (NTD). In [6]-[9], three-hop MIMO systems were addressed exploiting the structure of a Khatri-Rao space-time (KRST) coding to derive semi-blind receivers based on parallel factor (PARAFAC) models. However, there are still few results on tensor approaches for multi-hop scenarios.

In this paper, we propose a three-hop AF MIMO relay system with tensor coding at the source and the relays, which yields a received signals model that satisfies a fifth-order NTD

The authors would like to thank CAPES, CNPq and COFECUB for the financial support of this research. Danilo S. Rocha is supported by CAPES/PDSE/Process n ${ }^{\circ}$ 88881.135513/2016-01.
[5]. This system generalizes two other systems introduced in [5] and [6] by the use of links assisted by multiple relays and tensor coding, respectively. Considering the coding tensors known at destination, we propose an alternating least squares (ALS)-based semi-blind receiver to jointly estimate the symbols and the individual channels. We provide Monte Carlo simulation results to illustrate the impact of design parameters on the system performance and the behavior of the proposed receiver in terms of symbol-error-rate (SER), normalized mean square error (NMSE) of the estimated channels and speed of convergence. The simulation results show a significant performance improvement in the estimation of symbols and channels when compared with the estimation based on signals received with the direct link.

Notation: Scalars, column vectors, matrices and tensors of order higher than two are denoted by lowercase $(a, b, \cdots)$, boldface lowercase $(\mathbf{a}, \mathbf{b}, \cdots)$, boldface uppercase $(\mathbf{A}, \mathbf{B}, \cdots)$ and calligraphic $(\mathcal{A}, \mathcal{B}, \cdots)$ letters, respectively. $\mathbf{A}^{T}$ and $\mathbf{A}^{\dagger}$ denote the transpose and the Moore-Penrose pseudo-inverse of $\mathbf{A}$. Given a fourth-order tensor $\mathcal{X} \in \mathbb{C}^{I_{1} \times I_{2} \times I_{3} \times I_{4}}$, the third-order tensor $\mathcal{X}_{K L \times M \times N}$ is a contracted form obtained by combining two modes of $\mathcal{X}$, where $\{K, L, M, N\}$ is any permutation of $\left\{I_{1}, I_{2}, I_{3}, I_{4}\right\}$. The matrix $\mathbf{X}_{K L M \times N}$ denotes a tall unfolding of $\mathcal{X}$ whose the entries are $x_{k, l, m, n}=\left[\mathbf{X}_{K L M \times N}\right]_{(k-1) L M+(l-1) M+m, n}$. The Kronecker product is denoted by $\otimes$ and the operator vec $(\cdot)$ transforms a matrix into a column vector by stacking the columns of its matrix argument.

Given two tensors $\mathcal{A} \in \mathbb{C}^{I_{1} \times \cdots \times I_{\mathrm{N}}}$ and $\mathcal{B} \in \mathbb{C}^{J_{1} \times \cdots \times J_{\mathrm{M}}}$ such that the last mode of $\mathcal{A}$ is equal to the first mode of $\mathcal{B}$, i.e. $I_{\mathrm{N}}=$ $J_{1}$, we define the following contraction operation

$$
\mathcal{C}=\mathcal{A} \underline{x}_{N} \mathcal{B} \in \mathbb{C}^{I_{1} \times \cdots \times I_{N-1} \times J_{2} \times \cdots \times J_{M}}
$$

where the entries of the tensor $\mathcal{C}$ are given by

$$
c_{i_{1}, \cdots, i_{N-1}, j_{2}, \cdots, j_{M}}=\sum_{i_{N}=1}^{I_{N}} a_{i_{1}, \cdots, i_{N}} b_{i_{N}, j_{2}, \cdots, j_{M}}
$$

\section{THREE-HOP MIMO RELAY SYSTEM}

We consider a three-hop MIMO relay system composed of a source (S), two relays $\left(R_{1}\right.$ and $\left.R_{2}\right)$ and a destination (D), as illustrated in Fig. 1. $M_{k}$ denotes the number of antennas at node $k$, with $k \in\{0,1,2,3\}$, and the superscripts $r$ or $t$ are used for 


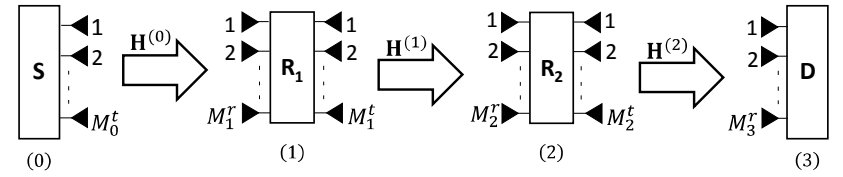

Fig. 1. Three-hop MIMO relay system.

receiving or transmitting antennas. The nodes indexed by $k=0$ and $k=3$ correspond to the source and the destination, respectively.

The transmission is carried out in three steps. In the first one, the source sends the coded data to $\mathrm{R}_{1}$. Then, in the second one, $\mathrm{R}_{1}$ transmits the received information to $\mathrm{R}_{2}$. Finally, $\mathrm{R}_{2}$ forwards the signals to the destination. In this transmission scheme, we make the following assumptions:

- the source and the relays encode the signals to be transmitted by means of a TSTC [3];

- the relays operate in half-duplex mode and use the AF protocol, retransmitting the received signals without decoding;

- $\quad$ synchronization is at the symbol level;

- all the channels are frequency-flat fading.

In the sequel, we describe the signal models for all the steps of transmission. Additive white Gaussian noises (AWGN) are omitted to simplify the presentation.

Let us define the symbol matrix $\mathbf{S} \in \mathbb{C}^{N \times R}$, where $R$ is the number of data streams transmitted at each symbol period $n$, with $n=1, \cdots, N$, where $N$ is the number of symbols per data stream. The coding tensor $\mathcal{C}^{(0)} \in \mathbb{C}^{M_{0}^{t} \times P_{0} \times R}$ used by the source leads to the coded signals $\chi^{(0)}=\mathcal{C}^{(0)} \times{ }_{3} \mathbf{S} \in \mathbb{C}^{M_{0}^{t} \times P_{0} \times N}$. Considering the channel matrix $\mathbf{H}^{(k)} \in \mathbb{C}^{M_{k+1}^{r} \times M_{k}^{t}}$ between the nodes $k$ and $k+1$, the tensor of signals received at the relay $\mathrm{R}_{1}$ satisfies the following Tucker model

$$
\begin{aligned}
\mathcal{X}^{(1)} & =\mathcal{X}^{(0)} \times_{1} \mathbf{H}^{(0)} \\
& =\mathcal{C}^{(0)} \times_{1} \mathbf{H}^{(0)} \times_{3} \mathbf{S} \in \mathbb{C}^{M_{1}^{r} \times P_{0} \times N} .
\end{aligned}
$$

The relays reencode the received signals by means of a coding tensor $\mathcal{C}^{(k)} \in \mathbb{C}^{M_{k}^{t} \times P_{k} \times M_{k}^{r}}$, for $k \in\{1,2\}$. The signals coded at the relay $R_{1}$ define the tensor $y^{(1)}=\mathcal{C}^{(1)} \times_{3} \chi^{(1)} \in$ $\mathbb{C}^{M_{1}^{t} \times P_{1} \times P_{0} \times N}$ to be transmitted to the relay $\mathrm{R}_{2}$. The signals received at the relay $R_{2}$ can be written as follows

$$
\begin{aligned}
\chi^{(2)} & =\mathcal{Y}^{(1)} \times_{1} \mathbf{H}^{(1)}=\left(\mathcal{C}^{(1)} \underline{\times}_{3} \chi^{(1)}\right) \times_{1} \mathbf{H}^{(1)} \\
& =\left(\mathcal{C}^{(1)} \times_{1} \mathbf{H}^{(1)}\right) \underline{\times}_{3} \mathcal{X}^{(1)} \in \mathbb{C}^{M_{2}^{r} \times P_{1} \times P_{0} \times N} .
\end{aligned}
$$

The tensor (4) satisfies a fourth-order NTD, or NTD(4), defined in [5]. Analogously, $\mathrm{R}_{2}$ encodes $\mathcal{X}^{(2)}$ and forwards the coded signals to the destination

$$
\chi^{(3)}=\left(\mathcal{C}^{(2)} \times_{1} \mathbf{H}^{(2)}\right) \underline{\times}_{3} \mathcal{X}^{(2)} \in \mathbb{C}^{M_{3}^{r} \times P_{2} \times P_{1} \times P_{0} \times N} .
$$

From the received signals (4)-(5), we define the following Tucker models

$$
\mathcal{T}^{(1)}=\mathcal{C}^{(1)} \times_{1} \mathbf{H}^{(1)} \in \mathbb{C}^{M_{2}^{r} \times P_{1} \times M_{1}^{r}}
$$

$$
\mathcal{T}^{(2)}=\mathcal{C}^{(2)} \times{ }_{1} \mathbf{H}^{(2)} \in \mathbb{C}^{M_{3}^{r} \times P_{2} \times M_{2}^{r}},
$$

and we rewrite the received signals tensor $\mathcal{X}^{(3)}$ as the following fifth-order NTD

$$
\chi^{(3)}=\mathcal{T}^{(2)} \underline{\times}_{3} \mathcal{T}^{(1)} \underline{x}_{3} \chi^{(1)} .
$$

The entries of the tensor $\mathcal{X}^{(3)}$ are given by

$$
\begin{gathered}
x_{m_{3}^{r}, p_{2}, p_{1}, p_{0}, n}^{(3)}=\sum_{m_{2}^{t}=1}^{M_{2}^{t}} \sum_{m_{2}^{r}=1}^{M_{2}^{r}} \sum_{m_{1}^{t}=1}^{M_{1}^{t}} \sum_{m_{1}^{r}=1}^{M_{1}^{r}} \sum_{m_{0}^{t}=1}^{M_{0}^{t}} \sum_{r=1}^{R} h_{m_{3}^{r}, m_{2}^{t}}^{(2)} \\
\cdot c_{m_{2}^{t}, p_{2}, m_{2}^{r}}^{(2)} h_{m_{2}^{r}, m_{1}^{t}}^{(1)} c_{m_{1}^{t}, p_{1}, m_{1}^{r}}^{(1)} h_{m_{1}^{r}, m_{0}^{t}}^{(0)} c_{m_{0}^{t}, p_{0}, r}^{(0)} S_{n, r} .
\end{gathered}
$$

\section{ALS RECEIVER}

In this section, we develop an ALS-based semi-blind receiver to estimate the symbols and the channel matrices.

\section{A. Three-hop system}

We now establish four matrix unfoldings of the tensor $\chi^{(3)}$ of signals received via relay-assisted link that will be used to derive the LS cost functions of the ALS algorithm for estimating the unknown matrices $\mathbf{H}^{(2)}, \mathbf{H}^{(1)}, \mathbf{H}^{(0)}$ and $\mathbf{S}$. We assume that the coding tensors $\mathcal{C}^{(2)}, \mathcal{C}^{(1)}$ and $\mathcal{C}^{(0)}$ are known at the destination.

From (8), we define the auxiliary tensors

$$
\begin{aligned}
& \mathcal{A}^{(1)}=\mathcal{T}^{(2)} \underline{\times}_{3} \mathcal{T}^{(1)} \in \mathbb{C}^{M_{3}^{r} \times P_{2} \times P_{1} \times M_{1}^{r},} \\
& \mathcal{A}^{(2)}=\mathcal{T}^{(1)} \underline{\times}_{3} \mathcal{X}^{(1)} \in \mathbb{C}^{M_{2}^{r} \times P_{1} \times P_{0} \times N},
\end{aligned}
$$

such that we can rewrite $\mathcal{X}^{(3)}$ as

$$
\chi^{(3)}=\mathcal{T}^{(2)} \underline{\times}_{3} \mathcal{A}^{(2)}=\mathcal{A}^{(1)} \underline{x}_{3} \chi^{(1)} .
$$

By combining some modes of $\mathcal{X}^{(3)}$, we define three contracted forms of $\mathcal{X}^{(3)}$ satisfying a Tucker-(2,3) model

$$
\begin{aligned}
X_{M_{3}^{r} P_{2} P_{1} \times P_{0} \times N}^{(3)} & =\mathcal{X}^{(1)} \times_{1} \mathbf{A}_{M_{3}^{r} P_{2} P_{1} \times M_{1}^{r}}^{(1)} \\
& =\mathcal{C}^{(0)} \times_{1} \mathbf{A}_{M_{3}^{r} P_{2} P_{1} \times M_{1}^{r}}^{(1)} \mathbf{H}^{(0)} \times_{3} \mathbf{S}, \\
\chi_{M_{3}^{r} P_{2} \times P_{1} \times P_{0} N}^{(3)} & =\mathcal{T}^{(1)} \times_{1} \mathbf{T}_{M_{3}^{r} P_{2} \times M_{2}^{r}}^{(2)} \times_{3} \mathbf{X}_{P_{0} N \times M_{1}^{r}}^{(1)} \\
& =\mathcal{C}^{(1)} \times_{1} \mathbf{T}_{M_{3}^{r} P_{2} \times M_{2}^{r}}^{(2)} \mathbf{H}^{(1)} \times_{3} \mathbf{X}_{P_{0} N \times M_{1}^{r}}^{(1)},
\end{aligned}
$$




$$
\begin{aligned}
X_{M_{3}^{r} \times P_{2} \times P_{1} P_{0} N}^{(3)} & =\mathcal{T}^{(2)} \times{ }_{3} \mathbf{A}_{P_{1} P_{0} N \times M_{2}^{r}}^{(2)} \\
& =\mathcal{C}^{(2)} \times_{1} \mathbf{H}^{(2)} \times_{3} \mathbf{A}_{P_{1} P_{0} N \times M_{2}^{r}}^{(2)},
\end{aligned}
$$

where $\mathbf{X}_{P_{0} N \times M_{1}^{r}}^{(1)}$ and $\mathbf{T}_{M_{3}^{r} P_{2} \times M_{2}^{r}}^{(2)}$ are matrix unfoldings of $\mathcal{X}^{(1)}$ and $\mathcal{T}^{(2)}$, and $\mathbf{A}_{M_{3}^{r} P_{2} P_{1} \times M_{1}^{r}}^{(1)}$ and $\mathbf{A}_{P_{1} P_{0} N \times M_{2}^{r}}^{(2)}$ are unfoldings of $\mathcal{A}^{(1)}$ and $\mathcal{A}^{(2)}$, given by

$$
\begin{gathered}
\mathbf{A}_{M_{3}^{r} P_{2} P_{1} \times M_{1}^{r}}^{(1)}=\left[\mathbf{T}_{M_{3}^{r} P_{2} \times M_{2}^{r}}^{(2)} \otimes \mathbf{I}_{P_{1}}\right] \mathbf{T}_{M_{2}^{r} P_{1} \times M_{1}^{r}}^{(1)}, \\
\mathbf{A}_{P_{1} P_{0} N \times M_{2}^{r}}^{(2)}=\left[\mathbf{I}_{P_{1}} \otimes \mathbf{X}_{P_{0} N \times M_{1}^{r}}^{(1)}\right] \mathbf{T}_{P_{1} M_{1}^{r} \times M_{2}^{r}}^{(1)} .
\end{gathered}
$$

Taking tall mode-1 unfoldings of (13)-(15) and a tall mode-3 unfolding of (13), we get respectively

$$
\begin{gathered}
\mathbf{X}_{P_{0} N \times M_{3}^{r} P_{2} P_{1}}^{(3)}=\left[\mathbf{I}_{P_{0}} \otimes \mathbf{S}\right] \mathbf{C}_{P_{0} R \times M_{0}^{t}}^{(0)}\left[\mathbf{A}_{M_{3}^{r} P_{2} P_{1} \times M_{1}^{r}}^{(1)} \mathbf{H}^{(0)}\right]^{T}, \\
\mathbf{X}_{P_{1} P_{0} N \times M_{3}^{r} P_{2}}^{(3)}=\left[\mathbf{I}_{P_{1}} \otimes \mathbf{X}_{P_{0} N \times M_{1}^{r}}^{(1)}\right] \mathbf{C}_{P_{1} M_{1}^{r} \times M_{1}^{t}}^{(1)}\left[\mathbf{T}_{M_{3}^{r} P_{2} \times M_{2}^{r}}^{(2)} \mathbf{H}^{(1)}\right]^{T},
\end{gathered}
$$

$$
\mathbf{X}_{P_{2} P_{1} P_{0} N \times M_{3}^{r}}^{(3)}=\left[\mathbf{I}_{P_{2}} \otimes \mathbf{A}_{P_{1} P_{0} N \times M_{2}^{r}}^{(2)}\right] \mathbf{C}_{P_{2} M_{2}^{r} \times M_{2}^{t}}^{(2)} \mathbf{H}^{(2)^{T}},
$$

$$
\mathbf{X}_{M_{3}^{r} P_{2} P_{1} P_{0} \times N}^{(3)}=\left[\mathbf{A}_{M_{3}^{r} P_{2} P_{1} \times M_{1}^{r}}^{(1)} \mathbf{H}^{(0)} \otimes \mathbf{I}_{P_{0}}\right] \mathbf{C}_{M_{0}^{t} P_{0} \times R}^{(0)} \mathbf{S}^{T}
$$

Equations (20)-(21) are useful to estimate $\mathbf{H}^{(2)}$ and $\mathbf{S}$, while (18)-(19) can be used to estimate $\mathbf{H}^{(0)}$ and $\mathbf{H}^{(1)}$ under vectorized form. Indeed, applying the property $\operatorname{vec}\left(\mathbf{A B C}^{T}\right)=(\mathbf{C} \otimes$ A) vec(B) to (18) and (19) gives

$$
\begin{aligned}
\mathbf{x}_{M_{3}^{r} P_{2} P_{1} P_{0} N}^{(3)}=\operatorname{vec} & \left(\mathbf{X}_{P_{0} N \times M_{3}^{r} P_{2} P_{1}}^{(3)}\right)=\left[\mathbf{A}_{M_{3}^{r} P_{2} P_{1} \times M_{1}^{r}}^{(1)} \otimes\right. \\
& \left.\left(\left(\mathbf{I}_{P_{0}} \otimes \mathbf{S}\right) \mathbf{C}_{P_{0} R \times M_{0}^{t}}^{(0)}\right)\right] \operatorname{vec}\left(\mathbf{H}^{(0)^{T}}\right),
\end{aligned}
$$

$$
\begin{array}{r}
\mathbf{x}_{M_{3}^{r} P_{2} P_{1} P_{0} N}^{(3)}=\operatorname{vec}\left(\mathbf{X}_{P_{1} P_{0} N \times M_{3}^{r} P_{2}}^{(3)}\right)=\left[\mathbf{T}_{M_{3}^{r} P_{2} \times M_{2}^{r}}^{(2)} \otimes\right. \\
\left.\left(\left(\mathbf{I}_{P_{1}} \otimes \mathbf{X}_{P_{0} N \times M_{1}^{r}}^{(1)}\right) \mathbf{C}_{P_{1} M_{1}^{r} \times M_{1}^{t}}^{(1)}\right)\right] \operatorname{vec}\left(\mathbf{H}^{(1)^{T}}\right) .
\end{array}
$$

Note that the vectorizations in (22)-(23) perform the same mode combinations of $\mathcal{X}^{(3)}$, thus producing the same vector.

The ALS receiver consists of alternately minimizing (in an iterative way) the LS cost functions derived from (20)-(23) with respect to $\mathbf{H}^{(2)}, \mathbf{S}, \mathbf{H}^{(0)}$ and $\mathbf{H}^{(1)}$, respectively. The algorithm is
TABLE I. ALS RECEIVER FOR RELAY-ASSISTED LINK

$$
\begin{aligned}
& \text { 1. Randomly initialize } \widehat{\mathbf{S}}_{i t=0}, \widehat{\mathbf{H}}_{i t=0}^{(1)} \text { and } \widehat{\mathbf{H}}_{i t=0}^{(0)} \text {. } \\
& \text { 2. } i t=i t+1 \text {. } \\
& \text { 3. Update the tensors } X_{(i t)}^{(1)}, \mathcal{T}_{(i t)}^{(1)} \text { and } \mathcal{A}_{(i t)}^{(2)} \text { using (3), (6) and (17). } \\
& \text { 4. Calculate the LS estimate of } \mathbf{H}^{(2)} \text { : } \\
& \left(\widehat{\mathbf{H}}_{i t}^{(2)}\right)^{T}=\left(\left[\mathbf{I}_{P_{2}} \otimes\left(\widehat{\mathbf{A}}_{P_{1} P_{0} N \times M_{2}^{r}}^{(2)}\right)_{i t}\right] \mathbf{C}_{P_{2} M_{2}^{r} \times M_{2}^{t}}^{(2)}\right)^{\dagger} \widetilde{\mathbf{X}}_{P_{2} P_{1} P_{0} N \times M_{3}^{r}}^{(3)} \\
& \text { 5. Update the tensors } \mathcal{T}_{(i t)}^{(2)} \text { and } \mathcal{A}_{(i t)}^{(1)} \text { using (7) and (16). } \\
& \text { 6. Calculate the LS estimate of } \mathbf{S}: \\
& \widehat{\mathbf{S}}_{i t}^{T}=\left(\left[\left(\widehat{\mathbf{A}}_{M_{3}^{r} P_{2} P_{1} \times M_{1}^{r}}^{(1)}\right)_{i t} \widehat{\mathbf{H}}_{i t-1}^{(0)} \otimes \mathbf{I}_{P_{0}}\right] \mathbf{C}_{M_{0}^{t} P_{0} \times R}^{(0)}\right)^{\dagger} \widetilde{\mathbf{X}}_{M_{3}^{r} P_{2} P_{1} P_{0} \times N}^{(3)} \\
& \text { 7. Calculate the LS estimate of } \mathbf{H}^{(0)}: \\
& \text { vec }\left(\widehat{\mathbf{H}}_{i t}^{(0)}\right)=\left[\left(\widehat{\mathbf{A}}_{M_{3}^{r} P_{2} P_{1} \times M_{1}^{r}}^{(1)}\right)_{i t} \otimes\left(\mathbf{I}_{P_{0}} \otimes \widehat{\mathbf{S}}_{i t}\right) \mathbf{C}_{P_{0} R \times M_{0}^{t}}^{(0)}\right]^{\dagger} \widetilde{\mathbf{x}}_{M_{3}^{r} P_{2} P_{1} P_{0} N}^{(3)} \\
& \text { 8. Update the tensor } X_{(i t)}^{(1)} \text { using (3). } \\
& \text { 9. Calculate the LS estimate of } \mathbf{H}^{(1)}: \\
& \text { vec }\left(\widehat{\mathbf{H}}_{i t}^{(1)}\right)= \\
& \left(\left(\widehat{\mathbf{T}}_{M_{3}^{r} P_{2} \times M_{2}^{r}}^{(2)}\right)_{i t} \otimes\left[\left[\mathbf{I}_{P_{1}} \otimes\left(\widehat{\mathbf{X}}_{P_{0} N \times M_{1}^{r}}^{(1)}\right)_{i t}\right] \mathbf{C}_{P_{1} M_{1}^{r} \times M_{1}^{t}}^{(1)}\right]\right)^{\dagger} \tilde{\mathbf{x}}_{M_{3}^{r} P_{2} P_{1} P_{0} N}^{(3)}
\end{aligned}
$$

10. Return to step 2 until convergence.

11. Eliminate the scaling ambiguities using (24)-(27).

12. Project the estimated symbols onto the symbol alphabet.

summarized in Table I, where it denotes the iteration number and the matrices $\widetilde{\mathbf{X}}_{P_{2} P_{1} P_{0} N \times M_{3}^{r}}^{(3)}, \widetilde{\mathbf{X}}_{M_{3}^{r} P_{2} P_{1} P_{0} \times N}^{(3)}$ and $\widetilde{\mathbf{x}}_{M_{3}^{r} P_{2} P_{1} P_{0} N}^{(3)}$ are noisy versions of $\mathbf{X}_{P_{2} P_{1} P_{0} N \times M_{3}^{r}}^{(3)}, \mathbf{X}_{M_{3}^{r} P_{2} P_{1} P_{0} \times N}^{(3)}$ and $\mathbf{x}_{M_{3}^{r} P_{2} P_{1} P_{0} N}^{(3)}$, respectively.

The uniqueness of NTD models was discussed in [5]. It is ensured by the knowledge of the core tensors $\left(\mathcal{C}^{(0)}, \mathcal{C}^{(1)}\right.$ and $\left.\mathcal{C}^{(2)}\right)$ at the destination, and the unknown factors are affected only by scaling ambiguities, i.e. $\overline{\mathbf{A}}=\alpha_{\mathbf{A}} \mathbf{A}$, where $\overline{\mathbf{A}}$ is an alternative solution for the matrix factor $\mathbf{A}$ to be estimated. These ambiguities are eliminated by assuming a priori knowledge of one pilot symbol and one channel coefficient in two of the three hops. We assume the knowledge of $s_{1,1}, h_{1,1}^{(1)}$ and $h_{1,1}^{(2)}$.

Thus, to eliminate the scaling ambiguities on the estimated parameters, we use the following equations

$$
\begin{aligned}
\widehat{\mathbf{S}} & \leftarrow \alpha_{\mathbf{S}} \widehat{\mathbf{S}} \\
\widehat{\mathbf{H}}^{(2)} & \leftarrow \alpha_{\mathbf{H}^{(2)}} \widehat{\mathbf{H}}^{(2)} \\
\widehat{\mathbf{H}}^{(1)} & \leftarrow \alpha_{\mathbf{H}^{(1)}} \widehat{\mathbf{H}}^{(1)} \\
\widehat{\mathbf{H}}^{(0)} & \leftarrow\left(\alpha_{\mathbf{S}} \alpha_{\mathbf{H}^{(2)}} \alpha_{\mathbf{H}^{(1)}}\right)^{-1} \widehat{\mathbf{H}}^{(0)},
\end{aligned}
$$

where $\alpha_{\mathbf{S}}=s_{1,1} / \hat{s}_{1,1}, \alpha_{\mathbf{H}^{(2)}}=h_{1,1}^{(2)} / \hat{h}_{1,1}^{(2)}$ and $\alpha_{\mathbf{H}^{(1)}}=h_{1,1}^{(1)} / \hat{h}_{1,1}^{(1)}$. 
The system identifiability depends on the uniqueness of the LS solutions. For computing the pseudo-inverses in Table I, their arguments must be left-invertible, i.e. they must be full column rank. This implies the following necessary conditions

$$
\begin{gathered}
P_{0} \geq \max \left(\frac{M_{0}^{t}}{R}, \frac{R}{M_{0}^{t}}, \frac{M_{1}^{r}}{N}\right), P_{1} \geq \max \left(\frac{M_{1}^{r}}{M_{2}^{r}}, \frac{M_{2}^{r}}{M_{1}^{r}}\right) \\
P_{2} \geq M_{2}^{r} / M_{3}^{r}, \quad M_{1}^{r} \geq M_{0}^{t}, \quad N \geq R .
\end{gathered}
$$

\section{B. One-hop system}

For the sake of comparison, we consider a one-hop system using TSTC at the source. In this subsection, we present this system and develop the corresponding ALS receiver to compare with the proposed three-hop system. We assume a one-hop MIMO system where the same encoded signals $x^{(0)}=$ $\mathcal{C}^{(0)} \times{ }_{3} \mathbf{S}$ are sent directly from the source to the destination through the channel $\mathbf{H}^{(0 \mapsto 3)} \in \mathbb{C}^{M_{3}^{r} \times M_{0}^{t}}$. The signals received at destination satisfy a Tucker-(2,3) model

$$
\chi^{(0 \mapsto 3)}=\mathcal{C}^{(0)} \times_{1} \mathbf{H}^{(0 \mapsto 3)} \times_{3} \mathbf{S} \in \mathbb{C}^{M_{3}^{r} \times P_{0} \times N},
$$

which can be written in scalar form as

$$
x_{m_{3}^{r}, p_{0}, n}^{(0 \mapsto 3)}=\sum_{m_{0}^{t}=1}^{M_{0}^{t}} \sum_{r=1}^{R} h_{m_{3}^{r}, m_{0}^{t}}^{(0 \mapsto 3)} c_{m_{0}^{t}, p_{0}, r}^{(0)} S_{n, r} .
$$

We consider tall mode-1 and -3 unfoldings of this Tucker model

$$
\begin{aligned}
& \mathbf{X}_{P_{0} N \times M_{3}^{r}}^{(0 \mapsto 3)}=\left[\mathbf{I}_{P_{0}} \otimes \mathbf{S}\right] \mathbf{C}_{P_{0} R \times M_{0}^{t}}^{(0)} \mathbf{H}^{(0 \mapsto 3)^{T}}, \\
& \mathbf{X}_{M_{3}^{r} P_{0} \times N}^{(0 \mapsto 3)}=\left[\mathbf{H}^{(0 \mapsto 3)} \otimes \mathbf{I}_{P_{0}}\right] \mathbf{C}_{M_{0}^{t} P_{0} \times R}^{(0)} \mathbf{S}^{T} .
\end{aligned}
$$

From these unfoldings, we propose a two-step ALS-based algorithm minimizing two LS cost functions built from (31)(32), with respect to $\mathbf{H}^{(0 \mapsto 3)}$ and $\mathbf{S}$, respectively. The ALS receiver for the direct link is summarized in Table II.

The uniqueness of the Tucker model is ensured by the knowledge of the core tensor $\mathcal{C}^{(0)}$ at the destination. The estimated parameters are affected by scaling ambiguities that can be eliminated by assuming a priori knowledge of one pilot symbol $\left(s_{1,1}\right)$. The ambiguities on the estimated parameters are eliminated using the following relations:

$$
\begin{gathered}
\widehat{\mathbf{S}} \leftarrow \alpha_{\mathbf{S}} \widehat{\mathbf{S}} \\
\widehat{\mathbf{H}}^{(0 \mapsto 3)} \leftarrow\left(\alpha_{\mathbf{S}}\right)^{-1} \widehat{\mathbf{H}}^{(0 \mapsto 3)},
\end{gathered}
$$

where $\alpha_{\mathbf{S}}=s_{1,1} / \hat{s}_{1,1}$.
TABLE II. ALS RECEIVER FOR DIRECT LINK

$$
\begin{aligned}
& \text { 1. Randomly initialize } \widehat{\mathbf{S}}_{i t=0} \text {. } \\
& \text { 2. it }=i t+1 \text {. } \\
& \text { 3. Calculate the LS estimate of } \mathbf{H}^{(0 \mapsto 3)} \text { : } \\
& \quad\left(\widehat{\mathbf{H}}_{i t}^{(0 \mapsto 3)}\right)^{T}=\left(\left[\mathbf{I}_{P_{0}} \otimes \widehat{\mathbf{S}}_{i t-1}\right] \mathbf{C}_{P_{0} R \times M_{0}^{t}}^{(0)}\right)^{\dagger} \widetilde{\mathbf{X}}_{P_{0} N \times M_{3}^{r}}^{(0 \mapsto 3)} \\
& \text { 4. Calculate the LS estimate of } \mathbf{S} \text { : } \\
& \quad \hat{\mathbf{S}}_{i t}^{T}=\left(\left[\widehat{\mathbf{H}}_{i t}^{(0 \mapsto 3)} \otimes \mathbf{I}_{P_{0}}\right] \mathbf{C}_{M_{0}^{t} P_{0} \times R}^{(0)}\right)^{\dagger} \widetilde{\mathbf{X}}_{M_{3}^{r} P_{0} \times N}^{(0 \mapsto 3)} \\
& \text { 5. Return to step } 2 \text { until convergence. } \\
& \text { 6. Eliminate the scaling ambiguities using (33)-(34). } \\
& \text { 7. Project the estimated symbols onto the symbol alphabet. }
\end{aligned}
$$

The left-invertibility of the pseudo-inverse arguments in the algorithm of Table II requires the following conditions:

$$
P_{0} \geq \max \left(\frac{M_{0}^{t}}{R}, \frac{R}{M_{0}^{t}}\right), \quad M_{3}^{r} \geq M_{0}^{t}, \quad N \geq R
$$

\section{SIMULATION RESULTS}

Monte Carlo simulations were performed to illustrate the behavior of the proposed receiver. The performance is evaluated in terms of symbol-error-rate (SER) and channel normalized mean square error (NMSE) versus symbol energy to noise spectral density ratio $\left(E_{S} / N_{0}\right)$.

The simulations results were averaged over $2.5 \times 10^{4}$ Monte Carlo runs. The transmitted symbols were randomly generated from a unit energy 4-QAM alphabet. The elements of the coding tensors have unit amplitude and random phase drawn from a uniform distribution between 0 and $2 \pi$. Each tensor $\mathcal{C}^{(k)}$ was multiplied by a fixed scalar gain so that the mean power at each transmission is controlled. Thus, the coding tensors become $\mathcal{C}^{(k)} \leftarrow \sqrt{\beta^{(k)}} \mathcal{C}^{(k)}$, for $k \in\{0,1,2\}$, with $\beta^{(k)}$ given by:

$$
\begin{gathered}
\beta^{(0)}=P_{T} / R M_{0}^{t}, \\
\beta^{(1)}=P_{T} / M_{1}^{r} M_{1}^{t}\left(P_{T} \alpha_{d}+N_{0}\right), \\
\beta^{(2)}=P_{T} / M_{2}^{r} M_{2}^{t}\left(P_{T} \alpha_{d}+N_{0}\right),
\end{gathered}
$$

where $P_{T}, \alpha_{d}$ and $N_{0}$ are the transmission power, channel attenuation and noise variance, respectively. For the three-hop system, we use $P_{T}=P_{\text {total }} / 3$, where $P_{\text {total }}$ is the power fixed for the system and arbitrarily chosen equal to 1 . In the case of the single-hop system, we keep the system total power and we assume $P_{T}=P_{\text {total }}$ at the source in order to compare the systems.

Additive white Gaussian noise tensors were simulated at each receiving node with the same noise variance $N_{0}$ for all nodes. At each Monte Carlo run, $N_{0}$ was fixed according to the desired $E_{S} / N_{0}$ value. 
The channels are assumed flat-fading and quasi-static, with i.i.d. (independent and identically distributed) complex Gaussian entries with unit variance. The channel attenuation $\alpha_{d}$ depends on the distance $d$ between two nodes, taking into account an exponential path-loss model, i.e. $\alpha_{d}=1 / d^{4}$. The distance $d$ (between relays, source and destination) was considered the same and equal to $D_{0} / 3$, where $D_{0}$ is the distance of the direct link (source-destination) arbitrarily chosen equal to 1 .

The impact of the choice of the design parameters was evaluated in the case of perfect channel knowledge using a zeroforcing (ZF) receiver, derived from step 6 of the algorithm in Table I and given by:

$$
\widehat{\mathbf{S}}^{T}=\left[\left[\mathbf{A}_{M_{3}^{r} P_{2} P_{1} \times M_{1}^{r}}^{(1)} \mathbf{H}^{(0)} \otimes \mathbf{I}_{P_{0}}\right] \mathbf{C}_{M_{0}^{t} P_{0} \times R}^{(0)}\right]^{\dagger} \widetilde{\mathbf{X}}_{M_{3}^{r} P_{2} P_{1} P_{0} \times N}^{(3)}
$$

For all the simulations, we have considered a same number of receive and transmit antennas at the relays, i.e. $M_{k}^{r}=M_{k}^{t}$. The design parameter values used in the simulations are indicated above each figure.

As a first result, we show the impact of the number $R$ of data streams on symbol estimation performance. Fig. 2 shows the SER versus $E_{S} / N_{0}$ for $R \in\{2,4,8\}$. As expected, one can note an increase of the SER when the value of $R$ is increased. However, a greater number of data streams improves the spectral efficiency by sending more symbols in the same time block.

Fig. 3 shows the SER versus $E_{S} / N_{0}$ for different timespreading lengths $P_{0}, P_{1}$ and $P_{2}$ of the coding tensors. The significant gain of the configuration $\left(P_{0}, P_{1}, P_{2}\right)=(4,2,2)$ shows that it is more efficient to have higher time-spreading at the nodes closest to the source. This result comes from the fact that the time-spreading at these nodes is repeated by all the nodes, improving the transmission.

Fig. 4 shows the SER versus $E_{S} / N_{0}$ for different numbers of antennas. One can note better results when increasing the number of antennas at the nodes closest to the source. These results corroborate the conclusions obtained in [5] concerning a greater efficiency in the exploitation of the space diversity at the source. This is due to the dependency of the coding tensors of each node with respect to the number of antennas. As expected, the case $(4,4,4,4)$ yields better performance than all the other cases, due to a greater number of antennas globally used at the source, the relays and the destination, while the case $(2,2,2,2)$ provides the biggest SER among the tested configurations.

The next results evaluate the performance of the proposed semi-blind ALS receiver. Fig. 5 shows the SER versus $E_{S} / N_{0}$ for the proposed receiver with the three-hop scenario (Table I) and for the ALS receiver of the direct link (Table II). One can conclude that the proposed three-hop system provides a significant gain over the estimation with the one-hop link. That comes from the multiple TST coding and from the fact that for the three-hop system the path-loss of each hop is smaller than the one of the single-hop system, due to the proportionality of the path-loss to $d^{4}$. For a fixed SER value $\left(10^{-3}\right)$, it can be observed that the $E_{S} / N_{0}$ gap is around

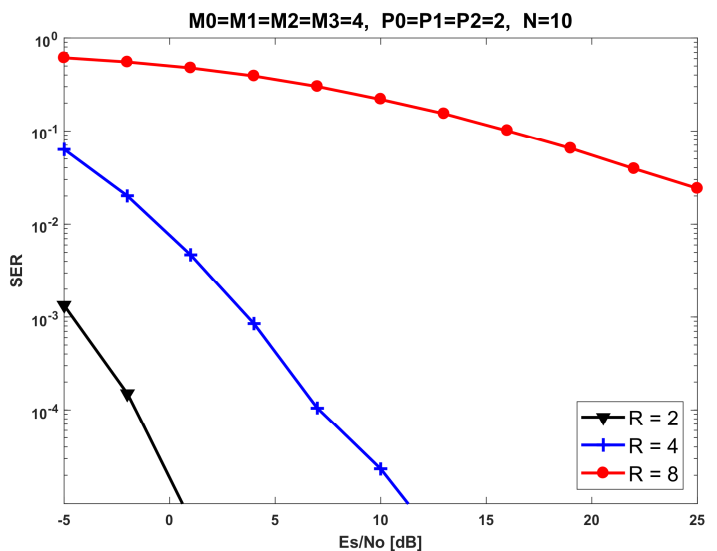

Fig. 2. ZF receiver performance for different numbers of data stream.

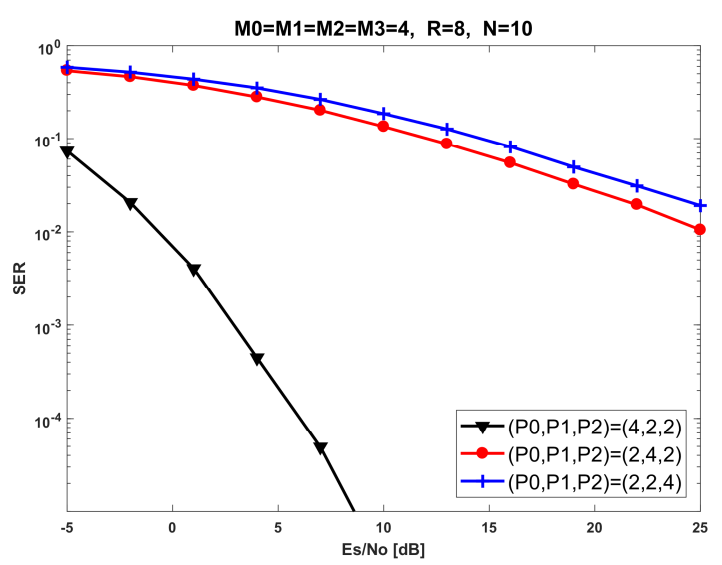

Fig. 3. ZF receiver performance for different values of $P_{0}, P_{1}$ and $P_{2}$.

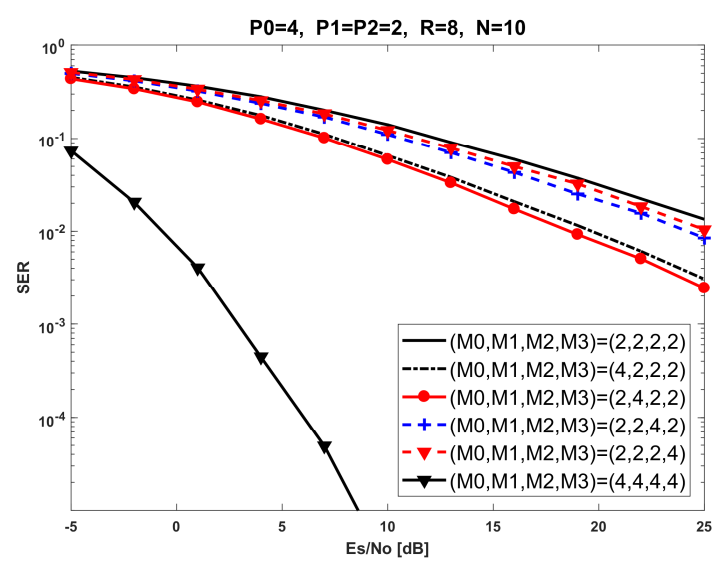

Fig. 4. ZF receiver performance for different numbers of antennas.

$5 \mathrm{~dB}$ and $20 \mathrm{~dB}$ for the ALS receivers of the three-hop and onehop systems, respectively, when compared with the $\mathrm{ZF}$ receiver. Despite this degradation, the proposed semi-blind ALS receiver has for advantages on the $\mathrm{ZF}$ receiver not to require a training sequence and also to allow a joint estimation of the symbols and the channels.

Fig. 6 shows the number of iterations versus $E_{S} / N_{0}$ needed to achieve the convergence with the iterative receivers. One can note the fastest convergence of the three-hop link for low $E_{S} / N_{0}$ 


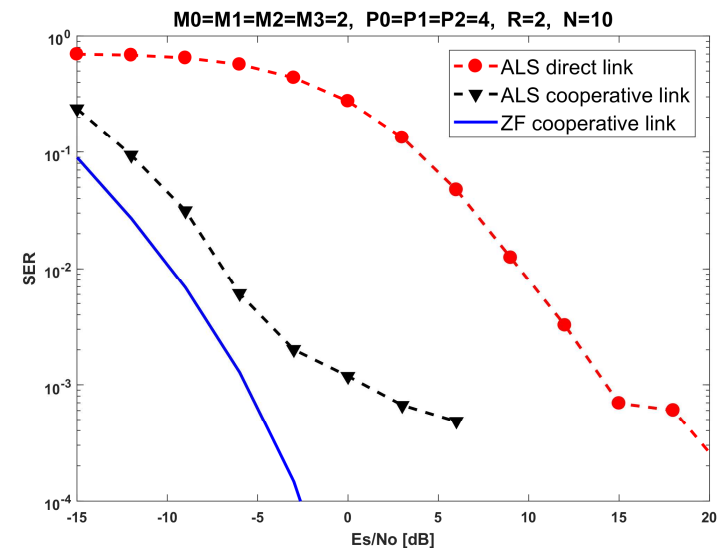

Fig. 5. SER performance for the proposed ALS receiver.

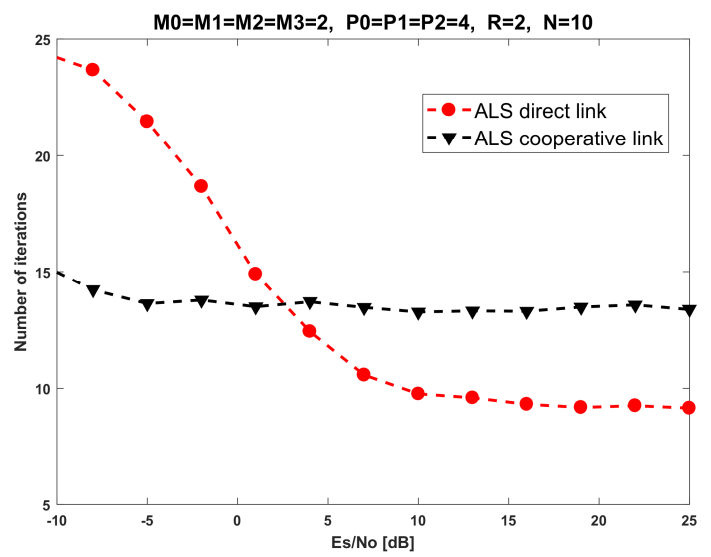

Fig. 6. Convergence of the proposed ALS receiver.

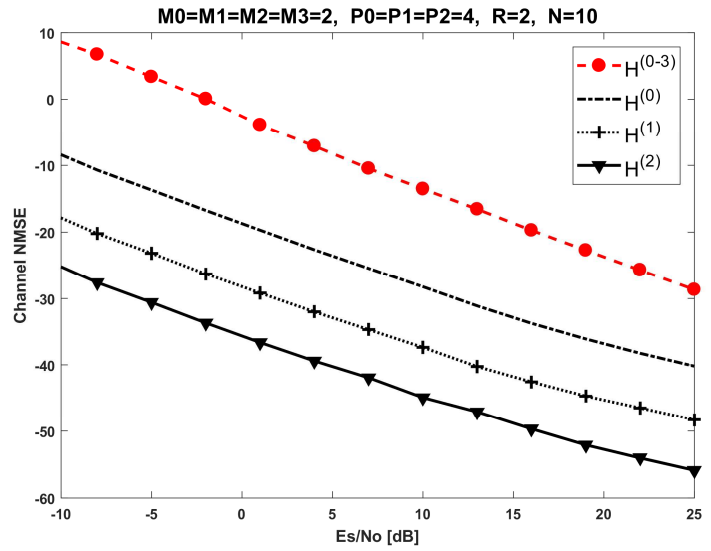

Fig. 7. Channel NMSE for the proposed ALS receiver.

values, showing the advantage of the cooperative network in an adverse situation. However, for high $E_{S} / N_{0}$ values, the smaller number of parameters to be estimated in the direct link increases the speed of convergence, overcoming the one obtained with the relay-assisted link.

In order to evaluate the estimation of the individual channels, Fig. 7 shows the channel NMSE versus $E_{S} / N_{0}$ for all the hops of the relay system. The NMSE has been computed as:

$$
N M S E=\frac{1}{M C} \sum_{m c}\left(\left\|\mathbf{H}_{m c}-\widehat{\mathbf{H}}_{m c}\right\|_{F}^{2} /\left\|\mathbf{H}_{m c}\right\|_{F}^{2}\right),
$$

with $m c=1, \cdots, M C, M C$ corresponding to the number of Monte Carlo runs, and $\|\cdot\|_{F}$ being the Frobenius norm. One can note that the channel estimation is improved for the nodes closest to the destination. Moreover, we can conclude that the channel estimation is better with the cooperative link than with the direct link.

\section{CONCLUSION}

We have proposed a three-hop AF MIMO relay system with tensor space-time coding (TSTC) at the source and the relays. The signals received at the destination form a fifth-order tensor that satisfies a nested Tucker decomposition. We have derived a semi-blind receiver based on a four-step ALS algorithm that jointly estimates the symbols and the channels. Simulation results have shown the performance of the proposed three-hop system is better than that of the direct link. Perspectives of this work include an extension to the case with $K>2$ relays and the development of a closed-form receiver.

\section{REFERENCES}

[1] N.D. Sidiropoulos, G.B. Giannakis and R. Bro, "Blind PARAFAC receivers for DS-CDMA systems," IEEE Trans. Signal Process., vol. 48, pp. 810-823, 2000.

[2] A.L.F. de Almeida, G. Favier, J.P.C.L. da Costa and J.C.M. Mota, "Overview of tensor decompositions with applications to communications," in Signals and Images: Advances and Results in Speech, Estimation, Compression, Recognition, Filtering, and Processing, CRC book, chapter 12, pp. 325-356, 2016.

[3] G. Favier, M.N. Costa, A.L.F. de Almeida and J.M.T. Romano, "Tensor space-time (TST) coding for MIMO wireless communication systems," Signal Process., vol. 92 (4), pp. 1079-1092, 2012.

[4] G.T. de Araújo and A.L.F. de Almeida, "Closed-form channel estimation for MIMO space-time coded systems using a fourth-order tensor-based receiver," Circuit Syst. Signal Process., 2017

[5] G. Favier, C.A.R. Fernandes and A.L.F. de Almeida, "Nested Tucker tensor decomposition with application to MIMO relay systems using tensor space-time coding (TSTC)," Signal Process., vol. 128, pp. 318$331,2016$.

[6] W.C. Freitas Jr., G. Favier and A.L.F. de Almeida, "Sequential closedform semi-blind receiver for space-time coded multi-hop relaying systems," IEEE Signal Process. Letters, vol. 24 (12), pp. 1773-1777, 2017.

[7] C.W.R. Chiong, Y. Rong, and Y. Xiang, "Channel training algorithms for two-way MIMO relay systems," IEEE Trans. Signal Process., vol. 61 (16), pp. 3988-3998, 2013.

[8] F. Gao, T. Cui, and A. Nallanathan, "On channel estimation and optimal training design for amplify and forward relay networks," IEEE Trans. Wireless Comm., vol. 7 (5), pp. 1907-1916, 2008.

[9] Y. Liu, S. Li, X. Mu and J. Zhang, "Tenor-based semi-blind channel estimation for three-hop MIMO relay systems," in Int. Conf. Wireless Comm. Signal Process. (WCSP), 2015.

[10] L.R. Ximenes, G. Favier, A.L.F. de Almeida, "Semi-blind receivers for non-regenerative cooperative MIMO communications based on nested PARAFAC modeling," IEEE Trans. Signal Process., vol. 63, pp. 49854998, 2015. 